\title{
Morbi-mortalidad en fracturas de húmero proximal tratadas quirúrgicamente
}




\section{ÍNDICE}

Introducción

5

Revisión y actualización bibliográfica

7

$\begin{array}{ll}\text { Anamnesis y exploración física } & 10\end{array}$

Tratamiento

11

Complicaciones de las fracturas de húmero proximal

Antecedentes patológicos

Hipótesis y objetivos

Material y métodos

Resultados

Discusión

Conclusiones

Bibliografía 


\section{INTRODUCCIÓN}

Las fracturas de fémur proximal son más frecuentes en personas con patología concomitante, encontrando una elevada morbi - mortalidad durante su seguimiento. Son la complicación más importante de la osteoporosis en términos de morbilidad, mortalidad y costes ${ }^{1}$.

Un $90 \%$ de los casos de fractura de cadera las encontramos en ancianos, un $78 \%$ de ellas en mujeres. Pacientes con ASA II o III, con anemia o limfocitopenia. Los niveles de albumina predicen la mortalidad hospitalaria. Aumenta la mortalidad en pacientes malnutridos. La edad media es superior a los 80 años.

Durante el primer año del postoperatorio, existe un aumento de riesgo de mortalidad entre un $14-36 \%$. Sobre todo en los primeros seis meses. El retraso de la intervención entre las 24 y 48 primeras horas no aumenta la mortalidad durante el primer año si, se ve aumentada, en cambio, si se retrasa más de 4 días, si necesita transfusiones o anestesia general ${ }^{2}$. En este tipo de fracturas encontramos un $5 \%$ de mortalidad hospitalaria afectando principalmente a personas de edad avanzada, de sexo masculino, en épocas de frío, especialmente en invierno. En el ámbito español el riesgo es mayor en Canarias y en comunidades del interior ${ }^{1}$.

Muchos de estos pacientes, después de la intervención quedan con secuelas y necesitan caminador.

En ancianos con fractura de cadera no se ha encontrado una diferencia estadísticamente significativa entre los que se les ha colocado una prótesis 
total de cadera, una fijación interna con reducción abierta o bien si se les ha realizado una hemiartroplastia teniendo en cuenta su patología de base ${ }^{3}$.

La mayoría de ellos son pacientes con ASA II o III, las mayores comorbilidades son enfermedades cardiovasculares, lesiones cerebrovasculares, enfermedades pulmonares, diabetes mellitus, artritis reumatoide y enfermedad renal $^{4}$.

Se han realizado numerosos estudios sobre los factores de riesgo en las fracturas de cadera, en mujeres, pero se ha investigado menos sobre la etiología de las fracturas de la extremidad superior, aun siendo las terceras fracturas más frecuentes en ancianos.

Un porcentaje igual a las fracturas de cadera lo conforman las fracturas de húmero proximal, de antebrazo y muñeca. Aunque estas tres modalidades incrementan modestamente la morbilidad del paciente, en comparación con las fracturas de cadera, que tienen considerables costes sobre la salud en pacientes relativamente jóvenes.

Una fuerza de prensión pobre también ha sido señalada como un factor de riesgo de fractura de cadera, al igual que, la debilidad del cuádriceps y la inestabilidad postural relacionadas con el aumento de las caídas. Esto indica no sólo una disminución de la densidad ósea, sino que también las caídas son un factor de riesgo alto, en las fracturas de húmero proximal, antebrazo y muñeca, especialmente en las mujeres ${ }^{5}$. 


\section{REVISIÓN Y ACTUALIZACIÓN BIBLIOGRÁFICA}

Las fracturas de húmero proximal representan un $5 \%$ de las lesiones del esqueleto ${ }^{6}$, siendo más frecuentes en el adulto seguidas de las de cadera y antebrazo, a partir de los 65 años. La mayoría son fracturas estables u osteoporóticas mínimamente desplazadas en caso de ancianos, afectando principalmente a mujeres, como resultado de caídas de poca energía.

Muchos de los pacientes con este tipo de lesiones recuperan la funcionalidad del hombro sin necesidad de intervención quirúrgica. La cirugía, sólo se considera en aproximadamente un $20 \%$ de los casos. En los jóvenes estas fracturas suelen ser producto de traumas violentos.

En 1970, Neer creó un sistema de clasificación de las fracturas de húmero proximal, que sigue siendo el más utilizado ${ }^{7}$.

Las fracturas abiertas de húmero proximal son poco frecuentes. Algunas muy desplazadas dejan la piel en tienda de campaña, causando necrosis por la presión ejercida. En estos casos se requiere tratamiento quirúrgico siguiendo las guías para fracturas abiertas.

En la fractura-luxación pueden aparecer en ocasiones lesiones vasculares. Los signos de isquemia distal están ausentes por su rica vascularización colateral, pero aparece un gran hematoma con un sangrado externo pulsátil o una hipotensión inexplicable con anemia ${ }^{8}$.

Muchas de las afectaciones del nervio son causadas por lesiones directas del plexo braquial o por tracción del nervio axilar, y más frecuentemente por 
fractura-luxación. La mayoría son cerradas y tratadas satisfactoriamente de manera conservadora ${ }^{6}$.

La estructura y disposición de las trabéculas óseas de la epífisis y de la metáfisis humeral permiten comprender los trazos de fractura más habituales, que suelen estar situadas por encima de la inserción del pectoral mayor.

Las fracturas pasan por las dos tuberosidades (mayor y menor), por el cuello anatómico y por el quirúrgico. Muchas de ellas son impactadas o desplazadas en abducción o aducción.

La importancia de estos cuatro fragmentos se encuentra en las fuerzas deformantes que actúan sobre los mismos una vez estos se fracturan, produciendo las alteraciones que a continuación se detallan:

- El manguito de los rotadores provoca un desplazamiento proximal y posterior de la tuberosidad mayor, el subescapular un alejamiento medial de la tuberosidad menor y la cabeza humeral.

- El pectoral mayor causa un desplazamiento medial de la diáfisis humeral.

- El deltoides abduce la diáfisis humeral ${ }^{8}$.

Las del cuello anatómico son poco habituales, salvo en caso de las fracturas luxaciones. La vascularización terminal de la cabeza humeral explica la gravedad de las mismas en pacientes de edad avanzada, donde con frecuencia se debe recurrir a la colocación de una prótesis.

En los niños, encontramos las fracturas en "tallo verde" de la metáfisis y las que comprenden desprendimientos epifisiarios. 
Las fracturas de húmero proximal son producidas por diferentes tipos de mecanismos, ya sean por traumatismos directos, por contusión sobre el hombro o indirectos, por caída sobre la mano o el codo, ya sea en abducción o en aducción. 


\section{Anamnesis y exploración física}

Independientemente de la edad del paciente, se debe realizar una detallada historia clínica acompañada de una exploración física. La anamnesis debe incluir el mecanismo de la lesión, la velocidad de la lesión (asociada a lesiones de costillas, cervicales o escapula), el nivel de funcionalidad, de ocupación, la mano dominante, historias de neoplasias, la habilidad para poder realizar rehabilitación funcional, etc.

La historia ha de incluir preguntas relacionadas con las parestesias del miembro afectado, la pérdida del conocimiento y si refiere dolor en codo o en la muñeca ipsilateral.

En la exploración física, debe examinarse el aspecto externo, buscando edema, lesión de los tejidos blandos, deformidad (visible en las fracturas en abducción) y más tarde equimosis difusa en brazo y codo.

La mayoría de pacientes acuden a urgencias con el brazo apoyado en el contralateral, en rotación interna. El intento de realizar movimientos pasivos o activos les produce un dolor severo y en la palpación observamos crepitación ${ }^{9}$.

Desde el inicio del examen debemos buscar las posibles complicaciones: vasculares, como la lesión de los vasos axilares (pulsos distales); nerviosas, como las afectaciones del plexo braquial, del nervio circunflejo, o del nervio radial; cutáneas, donde la solución de continuidad de la piel es rara; y por último las complicaciones músculo-tendinosas del deltoides y del tendón largo del bíceps (que puede impedir la reducción incruenta y justificar la cirugía). 


\section{Tratamiento}

Se ha observado una tendencia a tratar las fracturas de húmero proximal según la configuración del foco de la lesión. En el momento de la decisión se han de tener en cuenta las siguientes condiciones:

- Factores propios del paciente: muchos de los afectos por este tipo de lesiones son ancianos con limitadas expectativas de tratamiento. El quirúrgico no suele estar indicado en personas mayores de 85 años, con deterioro cognitivo, afectación previa de la extremidad fracturada o severas comorbilidades médicas.

En pacientes con severa osteoporosis, fumadores, drogadictos, alcohólicos, diabéticos, con artritis reumatoide, inmunodeprimidos por neoplasias o tratamiento con corticoides, se observan unos pobres resultados y un mayor riesgo de complicaciones.

- Factores propios del cirujano: el criterio del facultativo puede influenciar en los resultados dependiendo del tratamiento escogido y de su experiencia técnica.

- Factores propios de la lesión: las fracturas pueden ser clasificadas siguiendo diferentes criterios, la clasificación de Neer es una de las más utilizadas, a través de un sistema anatómico basado en el grado de participación y desplazamiento de cuatro segmentos descritos en las radiografías o en la cirugía.

En las fracturas de húmero proximal la cirugía es esencial, en las fracturas abiertas, las lesiones con afectación vascular, con estallido de la cabeza humeral, las que afectan a huesos patológicos y las que comportan una severa 
lesión del hombro ipsilateral frecuentemente causadas por fracturas de escapula con "hombro flotante".

En pacientes jóvenes y activos, una reducción con fijación permite obtener unos mejores resultados funcionales si los comparamos con el tratamiento conservador. La cirugía también reduce el riesgo de complicaciones asociadas al tratamiento no quirúrgico, incluyendo la pseudoartrosis y la consolidación viciosa sintomática, disminuyendo a la vez el riesgo de osteonecrosis de la cabeza femoral ${ }^{10}$.

Hemos de considerar la reducción y fijación de la fractura en:

- Fracturas con dos fragmentos de la tuberosidad mayor o menor, o fracturas de tres o cuatro, en que la tuberosidad mayor está desplazada más de un centímetro.

- Fracturas con un fragmento en la superficie articular de la cabeza humeral desplazado y con otro fragmento desplazado de la tuberosidad.

- Fracturas en dos partes del cuello quirúrgico inestables, con separación de la diáfisis humeral, respecto a la cabeza, por desplazamiento o conminución de la metáfisis.

- Fracturas de la cabeza humeral en dos, tres o cuatro fragmentos con deformidad en varo o valgo, en relación a la diáfisis con una angulación superior a 30 grados, respecto al ángulo de inclinación normal de 130 grados.

- Fractura - luxación anterior en tres o cuatro fragmentos con lesión de Hill - Sachs. 
- Fractura - luxación posterior en tres o cuatro fragmentos con lesión de Hill - Sachs inversa.

Se indica tratamiento quirúrgico en pacientes con oportunidad de mejorar la funcionalidad del hombro con respecto al tratamiento conservador ${ }^{6}$.

Las fracturas que se benefician del remplazo de la cabeza humeral son aquellas en que la reducción y la fijación son imposibles, donde la cabeza está escindida en dos o más fragmentos y es irreconstruible, en pacientes con pocas expectativas y poca funcionalidad, o después de un fracaso durante el seguimiento de una fractura tratada con reducción y fijación interna ya sea por pseudoartrosis o osteonecrosis de la cabeza.

En la planificación preoperatoria se precisan radiografías en proyección anteroposterior y transescapular lateral. En casos de difícil interpretación se debe realizar un TAC para planificar la intervención, calcular los grados de subluxación y angulación de la cabeza humeral, la separación de la diáfisis respecto a la cabeza, los grados de separación de los fragmentos marginales de las tuberosidades y la presencia de fragmentos intraarticulares, con el fin de confirmar el diagnóstico.

Teóricamente, en las fracturas de tres y cuatro fragmentos, la cabeza humeral recibe un subministro de sangre escaso, por lo que podemos encontrar necrosis avascular, con pérdida de funcionalidad del hombro ${ }^{11}$. 


\section{Tratamiento conservador}

El dolor de la lesión se controla habitualmente a partir de la primera semana con analgésicos orales y calor o frío local, la mayoría de los pacientes refieren dormir mejor sentados para el confort del dolor.

Los yesos colgantes no ofrecen ventajas, al distraer la fractura predisponen a la falta de consolidación o pseudoartrosis. No se aconseja la inmovilización prolongada, para empezar la rehabilitación rápidamente.

La mayoría de pacientes ancianos con fracturas estables o mínimamente desplazadas responden satisfactoriamente al tratamiento conservador, las tres mayores complicaciones son la pseudoartrosis, la consolidación viciosa y la osteonecrosis ${ }^{6}$. 


\section{Complicaciones de las fracturas de húmero proximal}

\section{Osteonecrosis}

Complicación inevitable de la lesión por falta de riego sanguíneo a nivel de la cabeza humeral, comúnmente en fracturas multifragmentarias y fracturasluxaciones. También como resultado de técnicas quirúrgicas pobres, con excesiva manipulación y afectación de tejidos blandos.

Se presenta con dolor, rigidez y pérdida de función, normalmente después de un período de correcta funcionalidad. Radiológicamente, los cambios varían desde una cabeza irregular con segmentos de esclerosis, a una completa reabsorción y colapso. La resonancia magnética permite evaluar la extensión y la severidad.

El núcleo de compresión puede ayudar en pacientes con cambios radiológicos rápidos, pero muchos acuden con sintomatología cuando ya padecen un colapso avanzado precisando un recambio de la cabeza humeral.

Pseudoartrosis

La pseudoartrosis de la cabeza - diáfisis humeral se presenta esporádicamente, suele asociarse a pacientes, a fracturas o a tratamientos con factores de riesgo.

Entre los factores de riesgo del paciente incluimos: osteoporosis, enfermedades inflamatorias o degenerativas del hombro, estado psicológico deteriorado, fumadores, drogadictos, alcohólicos y comorbilidades médicas. 
Las fracturas con más riesgo son las que no contactan la cortical entre la cabeza y la diáfisis del húmero o las conminutas. Cuando hay disrupción del periostio se interpone tejido blando y evita la formación de callo. Los yesos colgantes, la distracción de la fractura, una pobre técnica quirúrgica o mecanismos de reducción y fijación inestables causan pseudoartrosis.

Los pacientes acuden con severo dolor, rigidez y pérdida de función, generalmente con pseudoparalisis del deltoides, del manguito de los rotadores y músculos periescapulares. EI TAC nos confirma la pseudoartrosis. Si como primer tratamiento se realizó reducción abierta y fijación interna se ha de excluir la infección con aspiración guiada por ecografía.

\section{Consolidación viciosa}

Algunos grados de consolidación viciosa son inevitables en fracturas desplazadas de húmero proximal tratadas de forma conservadora. La consolidación viciosa de la cabeza - diáfisis y la de la cabeza - tuberosidades es frecuente y normalmente bien tolerada en pacientes ancianos.

\section{Rigidez de hombro postraumática}

Aunque la contractura capsular es la principal causa de rigidez, otros factores, incluida la consolidación viciosa de las fracturas, síndrome del complejo regional del dolor, los "impigments" de los implantes, la disfunción del manguito de lo rotadores son causa de rigidez a nivel del hombro.

El tratamiento inicial es conservador con rehabilitación y en aquellos pacientes con rigidez postraumática refractaria sin consolidación viciosa, se puede 
manipular bajo anestesia e incluso ayudar mediante la realización de una artroscopia realizando artrolisis del hombro.

Infección

Es una complicación poco frecuente, después de una reducción abierta y fijación interna, debido a una rica vascularización y cobertura de tejidos blandos. Las infecciones superficiales se solucionan comúnmente con antibioticoterapia. Las infecciones más profundas se tratan quirúrgicamente retirando el implante, desbridando la zona afectada y con prolongada terapia antibiótica. Es necesaria la retirada del material implantado, desbridamiento, inserción de un espaciador y terapia antibiótica endovenosa como tratamiento base para erradicar la infección ${ }^{9}$. 


\section{Antecedentes patológicos}

La OMS definió la osteoporosis en 1994, como una densidad mineral ósea (DMO) con valor menor de 2,5 desviaciones estándar a la de los jóvenes, el valor normal para adultos ( $T$ score $<-2,5$ ) representa el punto de referencia que se ha utilizado en la mayoría de los ensayos clínicos como importante criterio de inclusión y por consiguiente, sigue siendo de uso frecuente como umbral de intervención de una gran cantidad de profesionales.

Sin embargo, a pesar de que una baja DMO está fuertemente asociada con el riesgo de fractura, es sabido que los diferentes factores de riesgo, tales como la edad, la historia de una fractura por fragilidad previa y el uso de esteroides entre otros, aumentan el riesgo de fractura, siendo necesario mejorar la sensibilidad de la medición de la DMO para identificar pacientes con alto riesgo de fractura ${ }^{13}$.

De acuerdo con los datos estimados en sujetos mayores de 50 años en Europa en el año 2000, se sucedieron 62.000 nuevas fracturas de cadera, 575.000 de antebrazo y 250.000 de húmero proximal que han sido informadas, representando un $35 \%$ de las fracturas mundiales. Los costes directos de las fracturas osteoporóticas en Europa asciende alrededor de 36 billones de euros al año.

La principal causa clínica de la osteoporosis son las fracturas osteoporóticas, que están implicadas en el aumento de la morbimortalidad, afectando la calidad de vida de las personas que padecen esta enfermedad. Por este motivo se debe focalizar la atención a los pacientes con elevado riesgo de sufrir una fractura osteoporótica. 
Las fracturas osteoporóticas, se definen como aquellas que ocurren después de un impacto mínimo, producido por una caída desde una altura inferior a la del paciente sin inercia, sumada a la de los desplazamientos de sus pies al caminar.

Se ha comprobado que el grosor de la cortical de la porción distal del húmero, es un predictor fiable para detectar la osteoporosis generalizada, más incluso que la cortical del fémur o del metacarpiano.

Actualmente, encontramos escasa información acerca de los cambios producidos por la osteoporosis en el húmero proximal y su relación con el espesor de la cortical de la diáfisis humeral. Sería una técnica simple, permitiría determinar la calidad del hueso de la parte proximal del húmero para ayudar en la toma de decisiones quirúrgicas y en la selección de un método de reparación de la fractura.

Generalmente, después de una fractura sólo se realizan radiografías, por lo que sería interesante desarrollar una técnica radiográfica para la evaluación de la osteoporosis y el espesor de la cortical de la porción superior del húmero.

Algún estudio clínico ha investigado la densidad mineral ósea del húmero proximal de los pacientes, pero sólo logran medir la densidad del cuello quirúrgico, porque el acromion y la cavidad glenoidea se superponen a la cabeza humeral.

En caso de necesitarse otra prueba complementaria a la radiografía se realiza un TAC, aunque no proporciona la resolución necesaria para determinar la DMO del húmero. 
Se ha llegado a la conclusión, que el espesor de la cortical de la diáfisis del húmero proximal es un predictor de la densidad mineral ósea fiable y reproducible. Una cortical de un espesor de menos de $4 \mathrm{~mm}$ es altamente indicativa de una baja DMO. Por tanto, en estos pacientes, la fijación interna de las fracturas de húmero proximal se puede complicar, por lo que se recomendaría tratamiento conservador o la implantación de una prótesis de hombro.

Las directrices actuales hacen hincapié en la utilidad de la combinación de la DMO y el riesgo de factores clínicos para determinar el riesgo absoluto de fractura y decidir qué paciente han de ser tratados y que pacientes tienen un riesgo de fractura suficientemente bajo como para ser observados sin tratamiento.

Se ha observado que después de la edad y la DMO, otros tres factores a tener en cuenta son la paridad, antecedentes de fracturas y el tratamiento con THP (terapia hormonal postmenopáusica); esta última da lugar a una protección significativa contra las fracturas, las usuarias actuales de PHT tenían un riesgo 50 a 60\% más reducido de fracturas OP en comparación con las no usuarias.

Se ha comprobado que en comparación con las mujeres nulíparas, las mujeres con tres hijos o más tienen un $48 \%$ a $56 \%$ menos de riesgo de fractura por fragilidad, independientemente de la DMO y de otros factores de riesgo clínico.

Curiosamente existe una relación monótona entre el aumento de la paridad y el riesgo de fractura, que disminuye con el aumento de número de hijos. Esta relación ha sido reportada en la mayoría de estudios que examinaron la asociación entre la paridad y el riesgo de fractura ${ }^{14}$. 
La osteoporosis es una enfermedad asintomática hasta complicarse por una fractura del hueso sin traumatismo previo o después de un mínimo accidente. Es la enfermedad ósea más frecuente en humanos y representa un importante problema de salud en países desarrollados. La elevada incidencia de osteoporosis en el mundo y su mayor complicación, las fracturas osteoporóticas, también conocidas como fracturas por fragilidad, son reconocidas desde hace más de veinte años. Uno de los primeros metaanálisis de los riesgos de las fracturas fue publicado en 1996 demostrando la asociación entre la densidad mineral ósea y el riesgo de fracturas osteoporóticas.

La probabilidad de que una mujer con menopausia presente una fractura osteoporótica durante el resto de su vida (las más frecuentes son la vertebral, la de antebrazo, la de húmero y la de cadera) superan incluso el riesgo de sufrir cáncer de mama, con una probabilidad aproximada de un $40 \%$ mayor en países desarrollados y muy cerca del riesgo de padecer una enfermedad coronaria.

En cambio, un hombre de raza blanca de sesenta años tiene un $25 \%$ de riesgo de sufrir una fractura osteoporótica a lo largo de su vida. En realidad, la mortalidad post - fractura de cadera al año en un hombre es el doble que en una mujer.

La osteoporosis conduce al enrarecimiento de la estructura intrínseca del hueso. El adelgazamiento de la cortical con combinación de menos y más delgadas trabéculas que aportan inferiores propiedades mecánicas, por 
ejemplo, menor fuerza de compresión. Por todo ello es bien conocido el aumento de la incidencia de fracturas de menos energía ${ }^{15}$.

Desde hace más de veinte años los estudios han demostrado la importancia de las caídas en la incidencia de nuevas fracturas en sujetos con predisposición, estableciéndose una asociación entre el número de caídas y las fracturas. Ésta es más frecuente en pacientes de más de 75 años, que no la conocida asociación entre la osteoporosis y las fracturas.

Diferentes variables muestran el elevado riesgo de caídas como: un factor individual (historia anterior de caídas sucesivas, fuerza muscular de las extremidades inferiores, equilibrio o competencia postural, dificultades en la visión, empeoramiento cognitivo, diabetes insulino - dependiente, alcoholismo, bajo nivel de actividad física...), un factor ambiental (luces de casa, animales domésticos, alfombras,...) y factores iatrogénicos (diferentes grupos de medicamentos, combinación de medicamentos,...$)$.

La importancia de determinar la asociación entre el número de caídas y la aparición de una fractura por fragilidad, establece la posibilidad de su prevención, reduciendo así el número de caídas.

La elevada incidencia de caídas en ancianos (un 30\% de las personas mayores de 65 años se cae en casa cada año), asociada a la morbilidad y las pruebas disponibles, demuestran la relevancia de un estudio que ponga de manifiesto la asociación y determine la necesidad de prevenir los factores de riesgo. El número de caídas y el riesgo de fractura están influenciados por la dirección de la caída, la respuesta de protección a ella y la resistencia ósea del paciente ${ }^{16}$. 
Otro factor a tener en cuenta en las fracturas osteoporóticas es el uso de la fijación interna por el elevado número de fallos encontrados a causa de la debilidad del hueso fracturado.

En las fracturas de húmero proximal, el uso de placas de fijación no parece prevenir todas las complicaciones; encontramos hasta un $40 \%$ de fracturas con complicaciones descritas en los manuales. Por eso es interesante evaluar si la calidad del hueso afectado es un factor contribuyente importante.

Encontramos pocos estudios sobre los factores de riesgo de las fracturas de húmero proximal, estos refieren una baja densidad mineral ósea (influyendo las dietas bajas en calcio a partir de los 45 años), antecedentes de otras fracturas, antecedente materno de fractura de cadera, bajo nivel de actividad física, masa corporal baja (IMC) e incluso algunos refieren como factor contribuyente: brazo izquierdo dominante, depresión, epilepsia, medicación para las convulsiones y uso de aparatos auditivos. Aunque en otros estudios no se ha encontrado relación con un IMC bajo.

En la depresión encontramos un aumento de los niveles de interleucina-6, que estimulan los osteoclastos precursores, conduciendo a una mayor pérdida ósea.

Se ha observado que las mujeres tratadas con hormona posmenopáusica se encuentran más protegidas de sufrir una fractura de este tipo. Los varones presentan un menor riesgo, en comparación con las mujeres. Las personas de raza negra, son así mismo menos susceptibles de padecer fractura de húmero proximal que las de raza blanca ${ }^{17}$. 
Se ha estudiado la diabetes como factor de riesgo de fracturas específicas, asociado a una mayor densidad mineral ósea.

En comparación con los no diabéticos, las mujeres con diabetes que no precisan insulina tenían un mayor riesgo de fractura de cadera (riesgo relativo $[(R R), 1,82, I C$ del $95 \%$ intervalo de confianza (IC), 1,24-2,69] y de húmero proximal (RR, 1,94 del $95 \% \mathrm{Cl}, 1,24$ a 3,02) en los modelos multivariados controlados, como la edad, el índice de masa corporal, la densidad ósea, y otros factores asociados a las fracturas y la diabetes.

Los pacientes diabéticos tratados con insulina presentaban más del doble de riesgo a sufrir una fractura de pie (RR multivariado ajustado, 2,66, IC 95\%, 1,18 a 6,02) en comparación a los no diabéticos.

Se considera el tratamiento con insulina una marca de severidad de la diabetes. Se observa un mayor riesgo de fractura en pacientes insulino dependientes en todos los tipos de fractura, excepto la de cadera, pero la diferencia sólo es estadísticamente significativa en las fracturas de pie.

El tiempo de evolución de la diabetes, se ha asociado a un elevado riesgo de fractura, encontrando resultados estadísticamente significativos en las fracturas de cadera ${ }^{18}$.

Al estudiar si el calzado influyen en el riesgo de fracturas, se ha observado que los zapatos con tacones bajos y cubiertos, en caso de caída, permiten reducir el riesgo de fracturas en diferentes articulaciones. Con resultados significativos en los casos de fracturas de pie. 
Excepto en las fracturas de pelvis, se ha comprobado que el aumento de la actividad física durante el último año, se relaciona con una reducción del riesgo de fractura entre los pacientes que sufren caídas. Al aumentar el ejercicio mejora la movilidad, la fuerza muscular, el equilibrio, la densidad mineral ósea y el tiempo de reacción.

Las caídas en superficies duras aumentan la posibilidad de fractura de húmero proximal y de antebrazo ${ }^{19}$. 


\section{HIPÓTESIS}

Los pacientes afectos de fractura de húmero proximal tratados quirúrgicamente tienen menor morbi-mortalidad a medio plazo que aquellos que presentan una fractura del fémur proximal tratada quirúrgicamente.

\section{OBJETIVOS SECUNDARIOS}

1- Estudiar la dependencia social de los pacientes con fracturas del húmero proximal tratadas quirúrgicamente

2- Estudiar el número y tipo de fracturas posteriores a la fractura del húmero proximal.

3- Estudiar el número de pacientes diagnosticados de osteoporosis en el momento de la fractura de húmero proximal. 


\section{MATERIAL Y MÉTODOS}

Estudio retrospectivo de 97 pacientes del Hospital del Mar de Barcelona, tratados quirúrgicamente por presentar fractura del húmero proximal, encontramos 23 hombres y 74 mujeres con una media de 69 años de edad ( 28 - 89); 58 pacientes tenían afectado el hombro derecho y 39 pacientes el izquierdo. En el $86.5 \%$ de los casos era su brazo dominante.

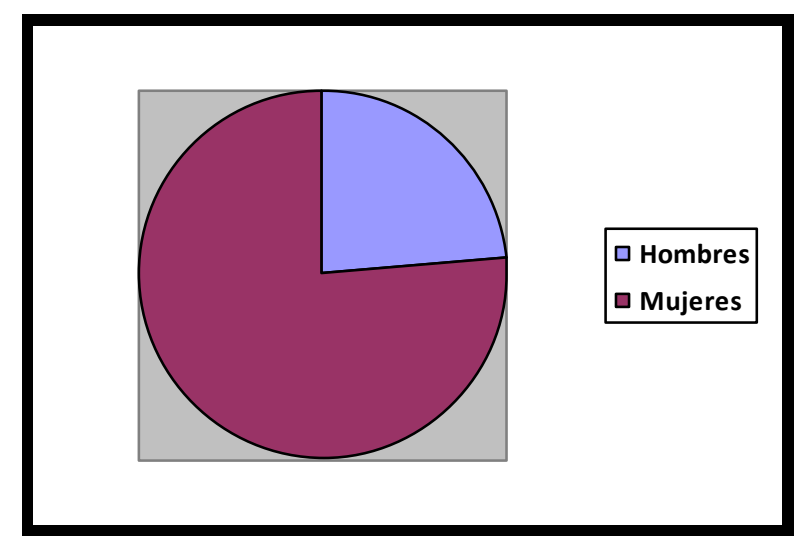

Todos ellos fueron diagnosticados de fractura de húmero proximal. Se clasificaron mediante la clasificación de Neer

Según Neer, la clasificación de las fracturas de húmero proximal hace referencia al nivel del foco de la fractura o al mecanismo de lesión y a la presencia o ausencia de desplazamiento de uno o más de los cuatro principales segmentos.

- Grupo I, mínimo desplazamiento.

Este grupo incluye todas las lesiones, con ningún segmento desplazado más de $1 \mathrm{~cm}$ o sin una angulación mayor a 45 grados. Lo constituyen el $85 \%$ de las fracturas de húmero proximal. Todas ellas presentan un tratamiento similar. Los fragmentos están recubiertos por tejidos blandos o están impactados. 
Se puede permitir una rápida movilización, aunque es necesario un período de inmovilización para evitar la rotación de la cabeza respecto a la diáfisis.

\section{- Grupo II, segmento articular desplazado.}

Puro desplazamiento del cuello anatómico sin separación de la tuberosidad. La lesión puede pasar inadvertida si no se realiza una correcta radiografía del húmero proximal en proyección anteroposterior, lo que puede dar lugar a una pseudoartrosis o necrosis avascular.

\section{- Grupo III, desplazamiento de la diáfisis.}

Esto ocurre distal a las tuberosidades, a nivel del cuello quirúrgico y con una separación de más de $1 \mathrm{~cm}$ o con una angulación de más de 45 grados. El manguito de los rotadores está integro.

Las fracturas epifisarias entran dentro de esta categoría. En los pacientes adultos se pueden diferenciar tres tipos:

- Fracturas de cuello quirúrgico anguladas e impactadas. Desviación residual de más de 45 grados causando una limitación permanente de la abducción y de la elevación.

- Fracturas desplazadas del cuello quirúrgico. Son aquellas en que la diáfisis se encuentra en dirección medial y anterior, hacia el pectoral mayor. El espacio entre los fragmentos aumenta con el brazo en abducción. Si se interpone tejido blando acaba produciéndose una pseudoartrosis. No es común que se asocie a lesión neurovascular. 
- Fracturas de cuello quirúrgico conminutas. Fragmentadas a nivel de la porción distal, con el brazo rotado internamente respecto al tórax. Los fragmentos intermedios se hallan desplazados hacia el pectoral.

- Grupo IV, desplazamiento de la tuberosidad mayor.

Su separación es patognomónica de lesión longitudinal del manguito de los rotadores, el supraespinoso. El fragmento fracturado es de pequeño tamaño, traccionado por la retracción muscular, lejos de su zona de inserción, debajo del acromio, visualizándose en la radiografía anteroposterior.

La tuberosidad mayor está sólidamente unida a tres músculos del manguito rotador por lo que la separación de los fragmentos es infrecuente. Éstos ejercen tracciones divergentes que se anulan entre sí, y confieren cierta estabilidad.

En fracturas con dos fragmentos, el segmento articular continua con una correcta relación respecto a la diáfisis, aunque se observa una mínima separación de la fractura a nivel del cuello quirúrgico. Normalmente una simple inmovilización en abducción moderada será suficiente.

En las de tres fragmentos, encontramos además de retracción de la tuberosidad, desplazamiento del cuello quirúrgico con el fragmento articular internamente rotado por el subescapular. Este tipo de fracturas tienen mejor pronóstico que las de cuatro fragmentos en que la cabeza está desplazada respecto a la diáfisis, suelen necesitar tratamiento quirúrgico. 
- Grupo V, desplazamiento de la tuberosidad menor.

Corresponde al arrancamiento de la tuberosidad, por la inserción del subescapular, en los movimientos contrarrestados de rotación externa forzada (deportes, accidentes de tráfico,...).

Las lesiones de dos fragmentos, formados por la avulsión de la tuberosidad menor o asociada a fractura no desplazada del cuello quirúrgico, separan la tuberosidad menor extendiendo las fibras anteriores del intervalo rotador causando una prominencia ósea.

En las fracturas de tres fragmentos desplazadas, el cuello quirúrgico se rota externamente, produciendo una abducción por acción del supraespinoso y de los rotadores externos.

En fracturas de cuatro fragmentos, las tuberosidades son retraídas con importante afectación vascular de la cabeza humeral, normalmente el fragmento articular se desplaza lateralmente entre ellas. Cuando la cabeza se lateraliza, pierde el contacto con la glenoides llamándose fractura-luxación lateral.

El diagnóstico se realiza mediante radiografía de perfil axilar.

El tratamiento ortopédico está indicado en las fracturas no desplazadas (inmovilización con un vendaje que mantenga el codo unido al cuerpo). El tratamiento quirúrgico, por su parte, está indicado en las fracturas desplazadas. 


\section{- Grupo VI, fractura-luxación.}

Éstas ocurren por luxaciones verdaderas con ruptura de los ligamentos y lesiones fuera de la articulación. El desplazamiento de la cabeza humeral es anteroinferior, posterior o superior. En las fracturas - luxaciones de dos y tres fragmentos normalmente se encuentran correctamente irrigados por la circulación que proviene de las tuberosidades.

La tuberosidad menor siempre queda unida a la cabeza humeral en las fracturas - luxación anterior de tres fragmentos mientras que la tuberosidad mayor es la que aporta la circulación a la cabeza humeral en las fracturas luxación posterior en tres fragmentos.

La fractura de la tuberosidad mayor asociada a una luxación antero - interna, se produce por traumatismos en hiperabducción del hombro, con un cizallamiento de la tuberosidad mayor, quedando atrapada entre la bóveda acromial y la glena. El fragmento de la tuberosidad mayor puede quedar prácticamente en contacto con el húmero, en su lugar normal, o completamente separado por la acción del infraespinoso.

Cuando el fragmento está poco desplazado, la reducción de la luxación permite el contacto entre la tuberosidad mayor y el húmero. La reducción puede ser perfecta si el húmero está en rotación neutra. A veces, la puesta en rotación interna (deseable después de una luxación antero-interna) es incompatible con una buena reducción de la tuberosidad mayor.

Cuando ésta permanece desplazada, debemos realizar una osteosíntesis. 
Las fracturas conminutas representan una problemática, porque existe riesgo de consolidación, y se forme una protuberancia que puede entrar en conflicto con el acromion y limitar la abducción.

Podemos encontrarnos delante de un caso particular, cuando la tuberosidad mayor conserva todas sus uniones normales con el manguito y su relación con el tendón del bíceps, mientras que la cabeza humeral se encuentra luxada.

Durante las tentativas de reducción, el tendón bicipital se opone a la reintegración de la cabeza humeral, atrapándola hacia atrás, lo que requerirá una reducción quirúrgica.

La afectación neurovascular sucede más frecuentemente en fracturas luxación anterior de cuatro fragmentos ${ }^{9}$. 


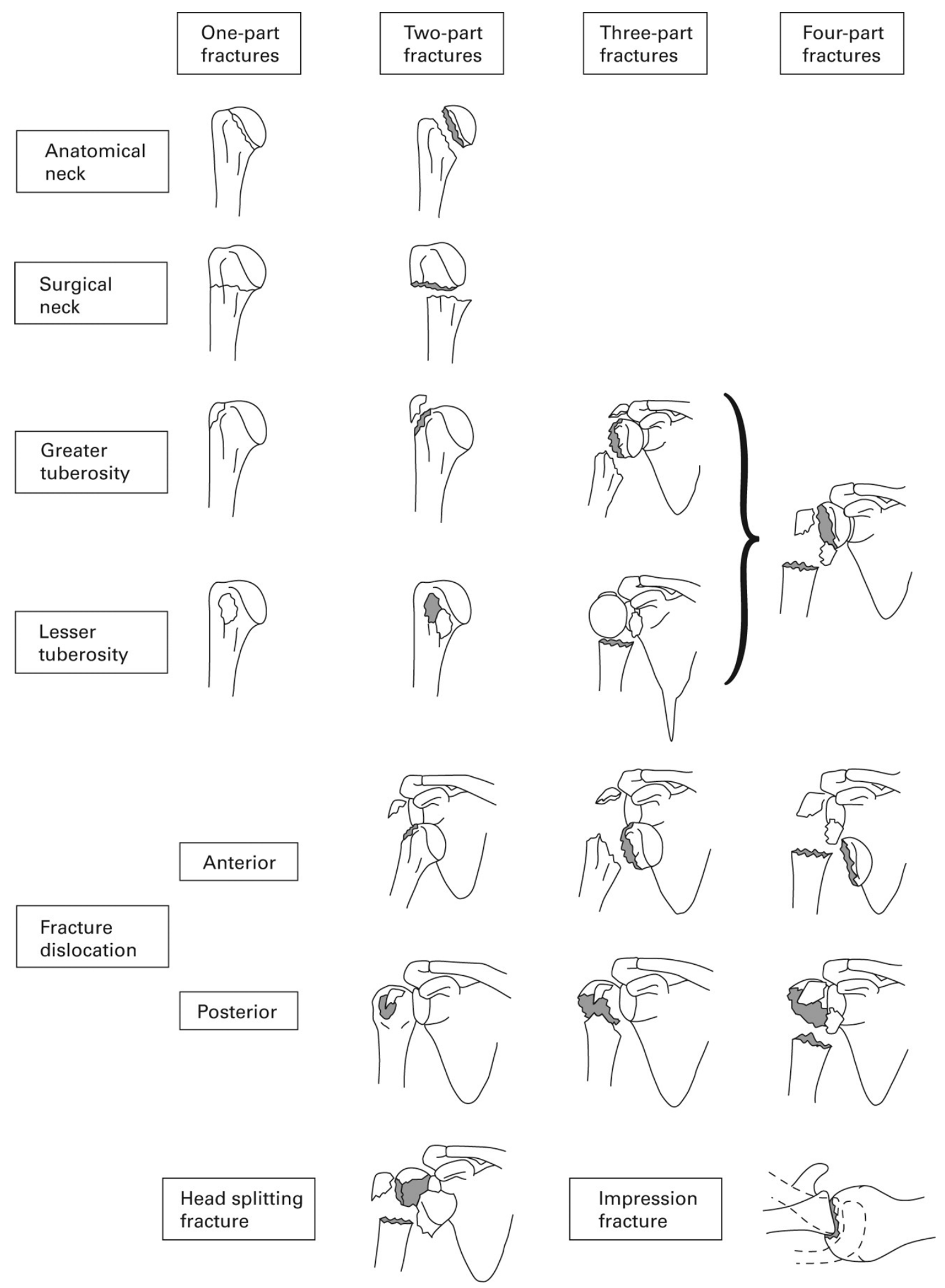

Fig 1. Clasificación de Neer 
En el estudio hemos encontrado:

27 fracturas en 2 fragmentos.

- 11 fracturas del cuello quirúrgico.

- 9 fracturas del cuello anatómico.

- 7 fracturas de la tuberosidad mayor.

43 fracturas en 3 fragmentos.

- 38 fracturas de la tuberosidad mayor.

- 5 fracturas de la tuberosidad menor.

19 fracturas en 4 fragmentos.

2 fractura - luxación en 2 fragmentos.

2 fractura - luxación en 3 fragmentos.

4 fractura - luxación en 4 fragmentos.

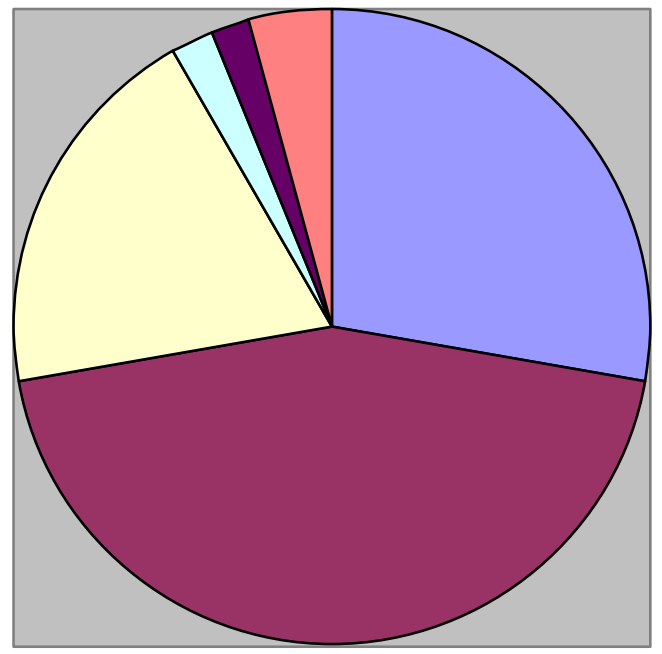

$\square$ Fr. en 2 fragmentos

$\square$ Fr. en 3 fragmentos

$\square$ Fr. en 4 fragmentos

$\square$ Fr - lux en 2 fragmentos

$\square \mathrm{Fr}$ - lux en 3 fragmentos

$\square$ Fr - lux en 4 fragmentos 
Los pacientes estudiados han sido objeto de diferentes tratamientos quirúrgicos dependiendo del tipo de fractura. De los cuales:

- 42 han sido intervenidos mediante suturas.

- 27 con sutura más enders.

- 4 con colocación de placa philos.

- 22 con hemiartroplastia.

- 2 con prótesis invertida.

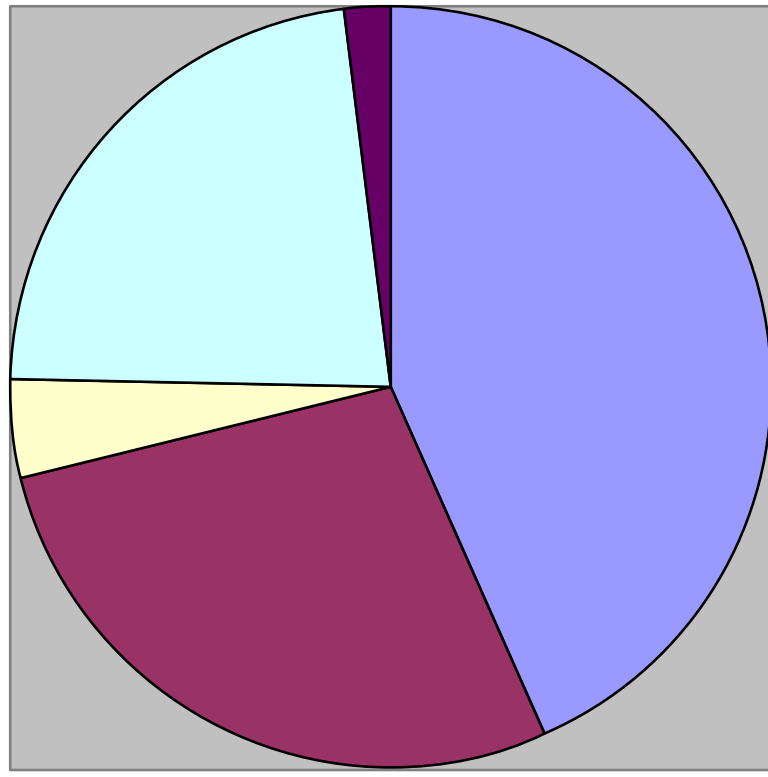

$\square$ Suturas

suturas + enders

$\square$ placa philos

$\square$ hemiartroplastia

prótesis invertida 


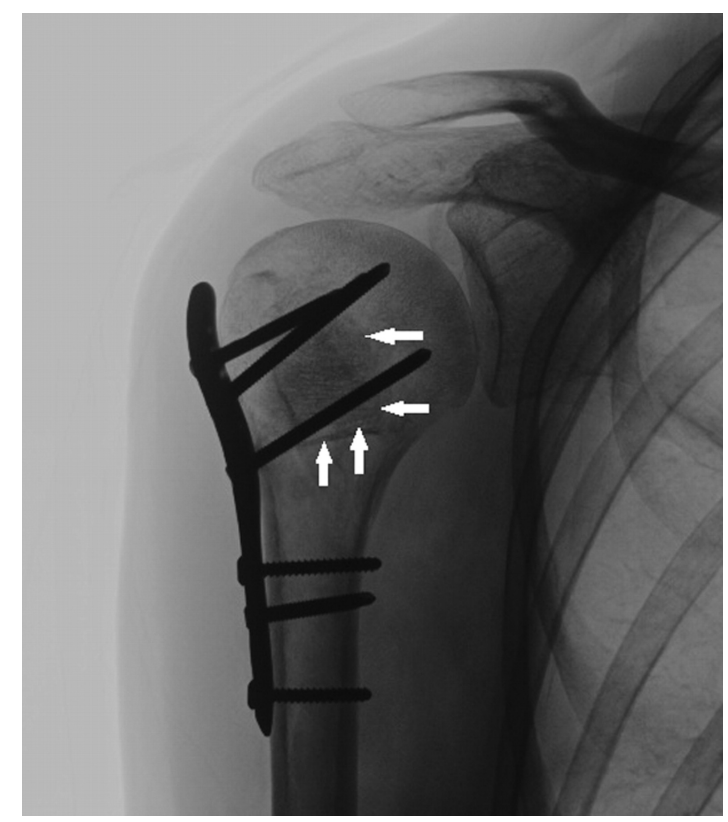

Fig 2. Fractura tratada con placa philos

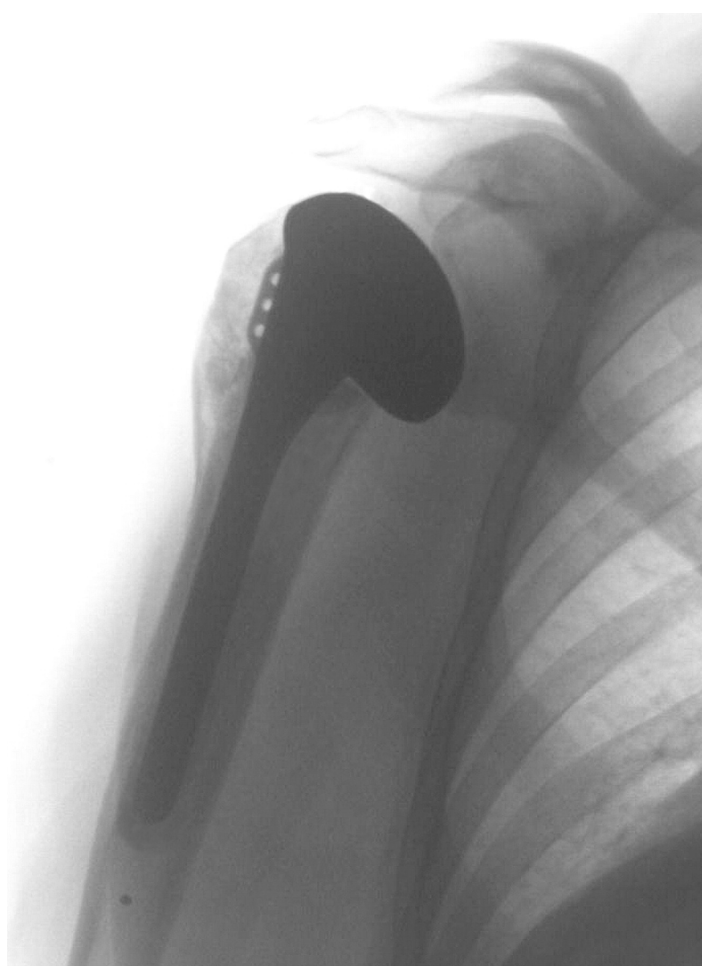

Fig 4. Fractura tratada con hemiartroplastia.

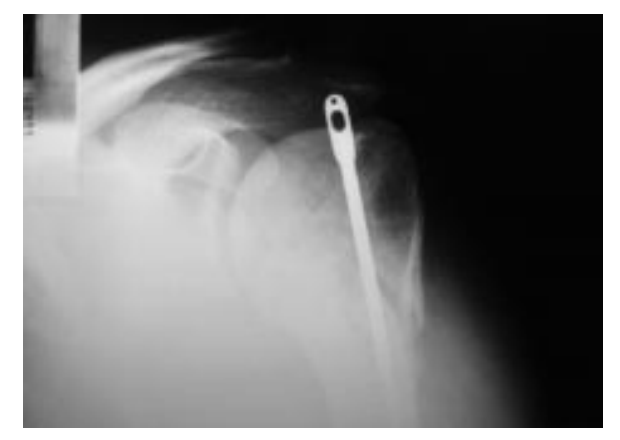

Fig 3. Fractura tratada con enders.

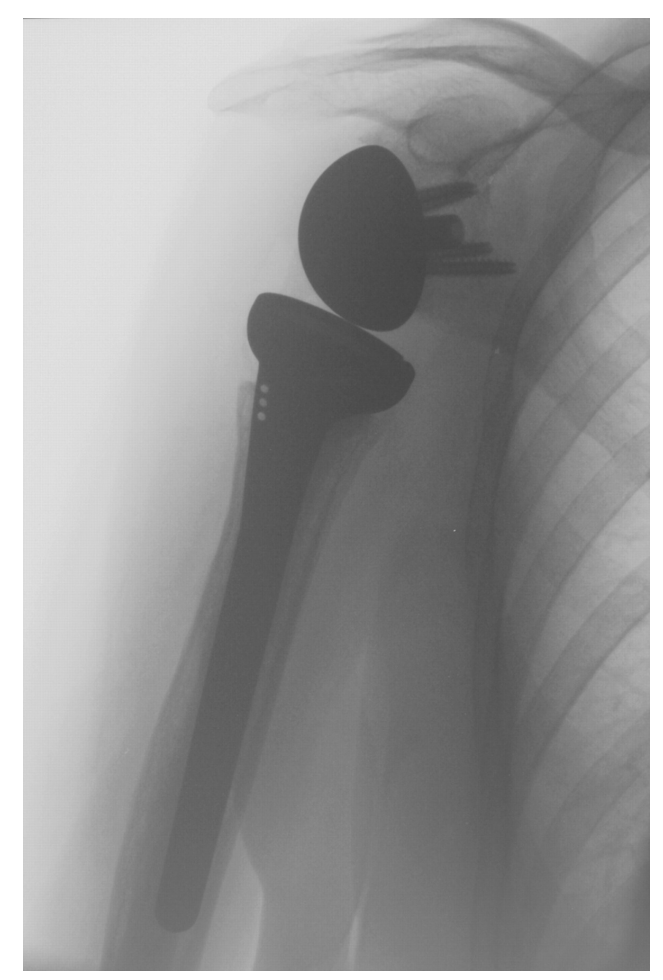

Fig 5. Fractura tratada con prótesis invertida. 
Se les siguió durante un período comprendido entre 1 y 12 años (siendo el seguimiento medio de 8 años), entre noviembre y diciembre del 2010 se les realizó una entrevista telefónica para determinar la morbi - mortalidad.

Se contabilizaron los pacientes que habían fallecido durante el seguimiento, si habían padecido fracturas posteriores a la fractura de húmero proximal, qué grado de satisfacción tenían de su hombro (muy satisfactorio, satisfactorio, descontento), si habían visto cumplidas sus expectativas (si, no), el grado de dependencia o independencia para las actividades de la vida cotidiana o si necesitaban ayuda para el aseo, las comidas,...

Se estudiaron los antecedentes personales (cardíacos, respiratorios, neurológicos y metabólicos) prestándose especial atención al hecho de padecer o no osteoporosis.

El estudio estadístico fue realizado con el programa informático SPSS. Windows 12.0. Usando La F-Fisher y la Chi-Cuadrado con un nivel de significación menor de 0.05 . 


\section{RESULTADOS}

De los 97 pacientes entrevistados, 22 no contestaron a la llamada, 75 respondieron a la misma y de estos en 14 la familia informó del fallecimiento del paciente, mientras que en 61 casos el paciente seguía vivo y pudo ser entrevistado.

De los 14 pacientes fallecidos, 12 de ellos son mujeres y 2 hombres. 9 tenían afectado el hombro derecho y 3 el izquierdo siendo 10 de ellos diestros y 2 zurdos.

En cuanto a la mortalidad, no se han encontrado diferencias estadísticamente significativas según las diferentes técnicas quirúrgicas. De todos modos queda reflejado que hay menor incidencia de defunciones en los pacientes tratados con sutura, mientras que aumenta el número de fallecidos en los tratados con sutura más ender.

Tampoco no se ha observado una relación estadísticamente significativa entre la mortalidad y el tipo de fractura.

\begin{tabular}{|l|l|l|}
\hline \multirow{2}{*}{ Tipo de fractura } & \multicolumn{2}{|l|}{ Exitus } \\
\cline { 2 - 3 } & SI & NO \\
\hline Fracturas en 2 partes & $12.5 \%$ & $87.5 \%$ \\
\hline Fracturas en 3 partes & $15.2 \%$ & $84.8 \%$ \\
\hline Fracturas en 4 partes & $21.4 \%$ & $78.6 \%$ \\
\hline Fractura - Luxación & $16.7 \%$ & $83.3 \%$ \\
\hline
\end{tabular}


Al relacionar la mortalidad con los antecedentes metabólicos, los cardíacos y la osteoporosis, se observa que los resultados serían significativos si aumentáramos el tamaño de la muestra, encontrando más fallecidos entre estos grupos.

No se contabilizó ningún fallecido entre los antecedentes respiratorios y solamente 2 entre los neurológicos. En cambio, observamos 4 y 13 exitus respectivamente entre los que padecían enfermedades cardíacas y metabólicas.

\begin{tabular}{|l|l|l|}
\hline Antecedentes & Mortalidad & Significación \\
\hline Cardíacos & $28.6 \%$ & 0.276 \\
\hline Respiratorios & $0 \%$ & 0.577 \\
\hline Neurológicos & $13.3 \%$ & 0.723 \\
\hline Metabólicos & & 0.171 \\
\hline
\end{tabular}

De los pacientes afectos de osteoporosis 4 de ellos también habían fallecido.

\begin{tabular}{|l|l|l|}
\hline \multirow{2}{*}{ Osteoporosis } & Mortalidad & Significación \\
\cline { 2 - 3 } & $36.4 \%$ & 0.111 \\
\hline
\end{tabular}

No se han encontrado diferencias estadísticamente significativas entre la edad del paciente y el tipo de fractura de húmero proximal. Por el contrario, si se han detectado diferencias estadísticamente significativas entre la edad y el tipo de 
intervención realizada, respecto las suturas, las suturas más enders y la prótesis.

\begin{tabular}{|l|l|l|l|}
\hline Tipo de & Media de edad & Desviación & \multirow{2}{*}{ Significación } \\
\hline Sutervención & 65.17 & 12.85 & \\
\hline Sutura + Ender & 71.16 & 12.89 & \multirow{2}{*}{0.003} \\
\hline Prótesis & 77.63 & 6.02 & \\
\hline
\end{tabular}

Los hombres padecen más fracturas de húmero de tres y cuatro fragmentos, observando que se les realizan un mayor número suturas aunque estas no sean estadísticamente significativas. En las mujeres las fracturas de húmero proximal suelen tener entre dos y tres fragmentos.

\begin{tabular}{|l|l|l|l|l|}
\hline \multirow{2}{*}{ Sexo } & \multicolumn{2}{|l|}{ Tipo de fractura } & \multirow{2}{*}{ Significación } \\
\cline { 2 - 4 } & 2 fragmentos & 3 fragmentos & 4 fragmentos & \multirow{2}{*}{0.476} \\
\hline Mujer & $25 \%$ & $51.6 \%$ & $23.4 \%$ & \\
\hline Hombre & $14.3 \%$ & $52.4 \%$ & $33.3 \%$ & \\
\hline
\end{tabular}

El sexo respecto al tipo de intervención, pone de manifiesto unos resultados estadísticamente significativos, con un elevado porcentaje de pacientes varones tratados con suturas, en cuanto a las pacientes del sexo femenino el resultado no difiere de forma relevante entre los casos tratados con sutura o con sutura y enders. 


\begin{tabular}{|l|l|l|l|l|}
\hline \multirow{2}{*}{ Sexo } & \multicolumn{2}{|l|}{ Tipo de cirugía } & \multirow{2}{*}{ Significación } \\
\cline { 2 - 5 } & Sutura & Sutura + & Prótesis & 0.036 \\
enders & & \\
\hline Mujer & $37.1 \%$ & $34.3 \%$ & $28.6 \%$ & \\
\hline Hombre & $72.2 \%$ & $16.7 \%$ & $11.1 \%$ & \\
\hline
\end{tabular}

Un $79.5 \%$ de los pacientes eran autónomos para las actividades de vida diaria (no necesitaban de ayuda externa de otras personas), con una edad media de 66,5 años.

Se les preguntó el grado de satisfacción del hombro intervenido quirúrgicamente, si estaban muy satisfechos del resultado de la intervención, satisfechos o descontentos, de los cuales un $59.4 \%$ (de los pacientes) evaluaron su hombro de manera muy satisfactoria, el $32.8 \%$ con un resultado satisfactorio y el $7.8 \%$ con mal resultado, ya que no habían visto cumplidas sus expectativas. Estos resultados han salido estadísticamente significativos respecto el grado de autonomía del paciente.

\begin{tabular}{|l|l|l|l|}
\hline \multirow{2}{*}{ ¿Cómo evaluaron } & \multicolumn{2}{|l|}{ Autónomo } & \multirow{2}{*}{ Significación } \\
\cline { 2 - 3 } & Sí & No & \multirow{2}{*}{0.003} \\
\hline Mal resultado & $33.3 \%$ & $66.7 \%$ & \\
\hline Satisfactorio & $68.2 \%$ & $31.8 \%$ & \\
\hline Muy satisfactorio & $90.2 \%$ & $9.8 \%$ & \\
\hline
\end{tabular}


Ante la pregunta que se les realizó sobre si su hombro les había quedado como esperaban después de la intervención, el 91.3\% respondió afirmativamente y un $8.7 \%$ negativamente, estos resultados son significativamente representativos respecto al grado de autonomía del paciente.

\begin{tabular}{|l|l|l|l|}
\hline \multirow{2}{*}{$\begin{array}{l}\text { ¿Les quedó como } \\
\text { esperaban? }\end{array}$} & \multicolumn{2}{|l|}{ Autónomo } & \multirow{2}{*}{ Significación } \\
\cline { 2 - 3 } & Sí & No & \multirow{2}{*}{0.018} \\
\hline Sí & $82.5 \%$ & $17.5 \%$ & \\
\hline No & $33.3 \%$ & $66.7 \%$ & \\
\hline
\end{tabular}

18 pacientes $(26 \%)$ sufrieron otras fracturas después de la fractura de húmero proximal, de estos un $61,1 \%$ continuaban siendo autónomos para sus actividades de la vida diaria con una relación estadísticamente significativa.

Así mismo, 9 pacientes padecieron fractura de cadera posteriormente a la de húmero, 4 fractura de columna, 5 fractura de radio distal, 2 fractura de clavícula, una fractura de tobillo, una de rótula, una del húmero contralateral y una de un metacarpiano.

\begin{tabular}{|l|l|l|l|}
\hline \multirow{2}{*}{ Fractura } & \multicolumn{2}{|l|}{ Autónomo } & Significación \\
\cline { 2 - 3 } & Sí & No & \multirow{2}{*}{0.05} \\
\hline Sí & $61.1 \%$ & $38.9 \%$ & \\
\hline No & $84.3 \%$ & $15.7 \%$ & \\
\hline
\end{tabular}


Se han correlacionado los antecedentes patológicos de los pacientes respecto a su autonomía, y no se ha encontrado relación estadísticamente significativa respecto a los antecedentes cardíacos, ni respiratorios, ni metabólicos. Si que se ha observado relación estadísticamente significativa con los antecedentes neurológicos, donde aparecen 13 pacientes afectos (17.5\%) de los cuales un $53,8 \%$ son autónomos para las actividades de la vida diaria.

\begin{tabular}{|l|l|}
\hline Antecedentes & Significación \\
\hline Cardiacos & 0.278 \\
\hline Respiratorios & 0.578 \\
\hline Neurológicos & 0.028 \\
\hline Metabólicos & 0.314 \\
\hline
\end{tabular}

Un $11.3 \%$ de los pacientes ya estaban diagnosticados de osteoporosis antes de la fractura de húmero proximal.

Al relacionar la autonomía del paciente con el hecho de haber sido diagnosticados de osteoporosis no se han observado unos resultados estadísticamente significativos. 9 pacientes (13\%) estaban afectos de ella.

\begin{tabular}{|l|l|l|l|}
\hline \multirow{2}{*}{ Osteoporosis } & \multicolumn{2}{|l|}{ Autónomo } & \multirow{2}{*}{ Significación } \\
\cline { 2 - 3 } & Sí & no & \multirow{2}{*}{0.672} \\
\hline Sí & $88.9 \%$ & $11.1 \%$ & \\
\hline No & $76.7 \%$ & $23.3 \%$ & \\
\hline
\end{tabular}


La relación entre el tipo de fractura de húmero proximal padecida y la autonomía del paciente no ha salido estadísticamente significativa.

Existe una relación estadísticamente significativa entre el tipo de intervención para estas fracturas y la autonomía posterior del paciente, de los 35 tratados mediante sutura, $32(91,4 \%)$ son autónomos, de los 17 pacientes operados mediante sutura más enders, 13 de ellos $(76,5 \%)$ son autónomos y de los intervenidos mediante placa philos y prótesis de hombro la mitad de continúan siendo autónomos para las actividades de la vida diaria.

\begin{tabular}{|l|l|l|l|}
\hline \multirow{2}{*}{ Tipo de cirugía } & \multicolumn{2}{|l|}{ Autónomo } & \multirow{2}{*}{ Significación } \\
\cline { 2 - 3 } & Sí & \multirow{2}{*}{0.006} \\
\hline Sutura & $91.4 \%$ & $8.6 \%$ & \\
\hline Sutura + enders & $76.5 \%$ & $23.5 \%$ & \\
\hline Prótesis & $50 \%$ & $50 \%$ & \\
\hline Philos & $50 \%$ & $50 \%$ & \\
\hline
\end{tabular}

Paralelamente se ha estudiado la relación entre la osteoporosis y si habían padecido algún otro tipo de fractura posteriormente a la fractura de húmero proximal o si había relación con el tipo de fractura de húmero encontrada.

De los 24 pacientes con fracturas posteriores a la de húmero, un 20,8\% sufrían osteoporosis sin relación estadísticamente significativa.

Dependiendo del tipo de fractura se observa que de los 19 pacientes con fracturas en 2 fragmentos un 5,3\% de ellas tenían osteoporosis, que de los 44 
con fracturas en 3 fragmentos un $11,4 \%$ de ellas tenían osteoporosis y de los

22 casos con fracturas en 4 fragmentos un $9,1 \%$ de ellas sufría de osteoporosis sin ser estadísticamente significativas ninguna de estas tres relaciones, por tanto, no se encuentra relación con el hecho de estar diagnosticado de osteoporosis y sufrir una fractura más conminuta o menos ni con el hecho de padecer más fracturas posteriormente a la fractura estudiada.

\begin{tabular}{|l|l|l|l|}
\hline \multirow{2}{*}{ Tipo de fractura } & \multicolumn{2}{|l|}{ Osteoporosis } & \multirow{2}{*}{ Significación } \\
\cline { 2 - 3 } & Sí & no & \multirow{2}{*}{0.455} \\
\hline 2 fragmentos & $5.3 \%$ & $94.7 \%$ & \\
\hline 3 fragmentos & $11.4 \%$ & $88.6 \%$ & \\
\hline 4 fragmentos & $9.1 \%$ & $90.9 \%$ & \\
\hline
\end{tabular}




\section{DISCUSIÓN}

La edad media de las fracturas de cadera en España está por encima de los 80 años, cada año aproximadamente unos 33.000 ancianos, de 82 años de media se fractura la cadera ${ }^{20}$; en cambio, en nuestro análisis encontramos una media de 66.5 años.

La mortalidad en las fracturas de fémur se sitúa entre un $14-36 \%$ durante el primer año postoperatorio, afectando a pacientes con patologías de base, la mayoría de ellos son clasificados como ASA II o ASA III. Afectando principalmente a varones ancianos ${ }^{2}$.

En nuestro estudio encontramos un $14.43 \%$ de exitus, la mayoría de ellos en mujeres ancianas, con un seguimiento medio de 8 años.

Queda bien establecido que las personas que han sufrido una fractura por fragilidad ósea tienen un riesgo significativamente mayor de tener un segunda en el futuro. Se ha observado que este riesgo disminuye con el tiempo. Los resultados sugieren que una fractura de húmero proximal puede ser predictiva de una fractura de cadera posterior.

En global, del $26 \%$ de pacientes con fracturas posteriores a la de hombro, un $61.1 \%$ de ellos continua siendo independiente para las actividades de la vida diaria. Se observan 9 pacientes con posterior fractura de cadera.

El mecanismo de producción de las fracturas de húmero proximal es similar al de las de cadera, teniendo en cuenta este hecho, es presumible que el espacio de tiempo transcurrido entre la fractura de húmero y la de cadera sea relativamente corto. 
Se ha observado en personas de edad avanzada, en general de ambos sexos, una probabilidad seis veces superior de sufrir fractura de cadera, posterior a una de húmero, siendo estadísticamente significativo dentro del primer año postraumático. No se han encontrado resultados significativos pasado el primer año. En otro estudio, el riesgo sigue siendo significativo durante los siguientes cinco años ${ }^{21}$.

No se ha encontrado relación estadísticamente significativa entre el aumento del número de caídas y las fracturas de antebrazo, tobillo, pie ${ }^{16}$.

En cuanto a sexos, es tres veces más frecuente en mujeres que en hombres, aumentando el riesgo exponencialmente con la edad.

De los pacientes diagnosticados de osteoporosis en el momento de la fractura de húmero proximal, observamos que un $11.3 \%$ de ellos la sufrían con anterioridad.

En nuestro trabajo hallamos un $79,5 \%$ de los pacientes que a los 8 años de media del tratamiento quirúrgico siguen siendo autónomos, para las actividades de la vida diaria siendo menor en aquellas personas diagnosticadas de osteoporosis o con fracturas posteriores a la fractura de húmero.

Un $92,2 \%$ de ellos están muy satisfechos o satisfechos del resultado obtenido mientras que un $91,3 \%$ han visto cumplidas sus expectativas, quedado el brazo como esperaban después de la intervención.

En el estudio hemos encontrado un $17.5 \%$ de los pacientes con antecedentes neurológicos y en los trabajos de fractura de cadera un $24.7 \%$. Un $16.7 \%$ con antecedentes cardíacos en comparación con un $26.8 \%$ en las fracturas de 
fémur proximal. Respecto a los antecedentes respiratorios, se observa un $6.2 \%$ en este trabajo y un $11.3 \%$ en las fracturas de cadera. En cuanto a antecedentes metabólicos observamos un $70.1 \%$ en equiparación a un $84.6 \%$ en los afectos de fractura de fémur proximal ${ }^{22}$.

Como queda de manifiesto en los resultados del presente estudio, los pacientes afectos de fractura de húmero proximal tratados quirúrgicamente tienen menor morbi - mortalidad a medio plazo que aquellos que presentan una fractura de fémur proximal tratada quirúrgicamente.

Por tanto, podríamos confirmar nuestra hipótesis nula al observar menos mortalidad en las fracturas de húmero proximal con un $14.43 \%$ con respecto hasta el $36 \%$ que encontramos en las fracturas de cadera. 


\section{CONCLUSIÓN}

\section{Conclusión de la hipótesis}

Por tanto, podríamos confirmar que observamos una menor mortalidad en las fracturas de húmero proximal con un $14.43 \%$ con respecto hasta el $36 \%$ que encontramos en las fracturas de cadera.

\section{Conclusión de los objetivos}

1- Al estudiar la dependencia social de los pacientes con fracturas de húmero proximal tratadas quirúrgicamente se ha verificado que sólo el $20.5 \%$ de ellos a los 8 años de seguimiento son dependientes para las actividades de la vida diaria.

2- Al analizar el número y tipo de fracturas posteriores a la fractura de húmero proximal detectamos 18 personas que padecieron fractura de: cadera, columna, radio distal, clavícula, tobillo, rótula, húmero contralateral y metacarpiano en orden de frecuencia de mayor a menor, con posterioridad a la fractura de húmero proximal estudiada.

3- Al investigar el número de pacientes diagnosticados de osteoporosis en el momento de la fractura de húmero proximal, observamos que un $11.3 \%$ de ellos la sufrían con anterioridad. 


\section{BIBLIOGRAFÍA}

1- Serra J,A; Garrido G; Vidán M; et al. Epidemiology of hip fractures in the elderly in Spain. An Med Interna. 2002 Aug;19(8):389-95.

2- Oztürk A; Ozkan Y; Akgöz S; et al. The risk factors for mortality in elderly patients with hip fractures: postoperative one-year results. Singapore Med J. 2010 Feb;51(2):137-43.

3- Radcliff T.A; Henderson W.G; Stoner T.J; et al. Patient risk factors, operative care, and outcomes among older community-dwelling male veterans with hip fracture. J Bone Joint Surg Am. 2008 Jan;90(1):34-42.

4- Ekström W; Miedel R; Ponzer S.J; et al. Quality of life after a stable trochanteric fracture. A prospective cohort study on 148 patients. Orthop Trauma. 2009 Jan;23(1):39-44.

5- Nguyen, T.V.; Center J.R.; Sambrook P.N.; Eisman J.A. Risk factors for proximal humerus, forearm, and wrist fractures in elderly men and women. The Dubbo osteoporosis epidemiology study. American Journal of Epidemiology. 2001 Mar 15;153(6):587-95.

6- Murray I.R; Amin A.K; White T.O; et al. Proximal humeral fractures: current concepts in classification, treatment and outcomes. J Bone Joint Surg Br. 2011 Jan;93(1):1-11.

7- Rockwood C.A; Matsen F.A; Wirth M.A; et al. The Shoulder. 4ed. Philadelphia. Saunders, 2009; 9: 295-332.

8- McLaurin T.M. Proximal humerus fractures in the elderly are we operating on too many? Bull Hosp Jt Dis. 2004;62(1-2):24-32. 
9- Habermeyer P; Magosch P; Lichtenberg S. Classifications and scores of the shoulder. Heilderberg. Springer, 2006; 11: 119-131.

10-Tamai K; Ishige N; Kuroda S. Four-segment classification of proximal humeral fractures revisited: a multicenter study on 509 cases. J Shoulder Elbow Surg. 2009 Nov-Dec;18(6):845-50. Epub 2009 Mar 17.

11-den Hartog D; de Haan J; Schep N.W; et al.Primary shoulder arthroplasty versus conservative treatment for comminuted proximal humeral fractures: a systematic literature review. Open Orthop J. 2010 Feb 17;4:87-92.

12-Baumgartner D; Nolan B.M; Mathys R; et al. Review of Fixation Techniques for the Four-part Fractured Proximal Humerus in Hemiarthroplasty. J Orthop Surg Res. 2011 Jul 18;6(1):36.

13-Tingart M.J; Apreleva M; von Stechow D; et al. The cortical thickness of the proximal humeral diaphysis predicts bone mineral density of the proximal humerus. J Bone Joint Surg Br. 2003 May;85(4):611-7.

14-Trémollieres F.A; Pouillès J.M; Drewniak N; et al. Fracture risk prediction using BMD and clinical risk factors in early postmenopausal women: sensitivity of the WHO FRAX tool. J Bone Miner Res. 2010 May;25(5):10029.

15-Azagra R; Roca G; Encabo G; et al. Prediction of absolute risk of fragility fracture at 10 years in a Spanish population: validation of the WHO FRAX TM tool in Spain. BMC Musculoskelet Disord. 2011 Jan 28;12:30.

16-Schwartz A.V; Sellmeyer D.E; Ensrud K.E, et al. Older women with diabetes have an increased risk of fracture: a prospective study. J. Clin. Endocrinol. Metab. 2001 Jan;86(1):32-8. 
17-Chu S.P; Kelsey J.L; Keegan T.H; et al. Risk factors for proximal humerus fracture. Am J Epidemiol. 2004 Aug 15;160(4):360-7.

18-Schwartz A.V; Nevitt M.C; Brown B.W. Jr. et al. Increased falling as a risk factor for fracture among older women: the study of osteoporotic fractures. Am J Epidemiol. 2005 Jan 15;161(2):180-5.

19-Keegan T.H; Kelsey J.L; King A.C; et al. Characteristics of fallers who fracture at the foot, distal forearm, proximal humerus, pelvis, and shaft of the tibia/fibula compared with fallers who do not fracture. Am J Epidemiol. 2004 Jan 15;159(2):192-203.

20-Beringer T.R; Gilmore D.H; Outcome following proximal femoral fracture in the elderly female. Ulster Med J. 1991 Apr;60(1):28-34.

21-Clinton J; Franta A; Polissar N.L; et al. Proximal humeral fracture as a risk factor for subsequent hip fractures. J Bone Joint Surg Am. 2009 Mar $1 ; 91(3): 503-11$.

22-Angthong C; Suntharapa T; Harnroongroj T. Major risk factors for the second contralateral hip fracture in the elderly. Acta Orthop Traumatol Turc. 2009 May-Jul;43(3):193-8. 